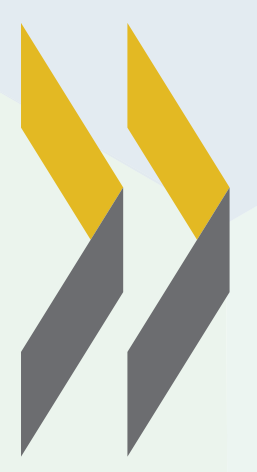

\title{
Comment l'enquête PISA mesure-t-elle les compétences de collaboration des élèves ?
}

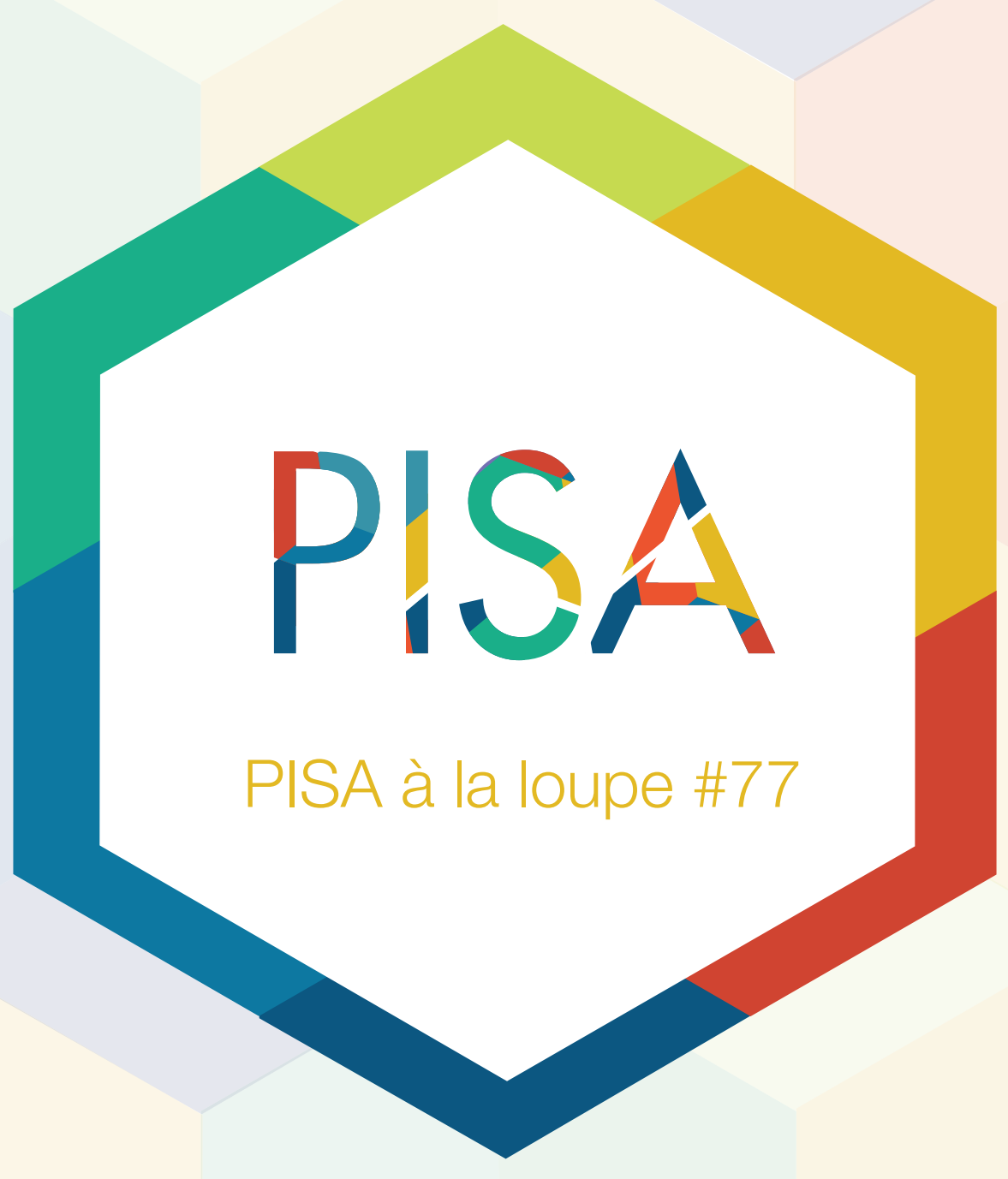



des élèves ?

- Les résultats de l'évaluation PISA 2015 de la résolution collaborative de problèmes seront publiés le 21 novembre 2017.

- II s'agit de la première évaluation internationale de la capacité des élèves à collaborer avec d'autres pour résoudre des problèmes.

- Environ 125000 élèves de 52 pays et économies y ont participé.

Tous les trois ans, l'enquête PISA évalue les compétences des élèves en sciences, en compréhension de l'écrit et en mathématiques. La maîtrise des fondamentaux à l'école et l'application de ces connaissances à des contextes familiers ne suffisent toutefois pas pour réussir dans la vie. Même en l'absence de connaissances spécifiques ou de procédures familières sur lesquelles s'appuyer, les élèves doivent être capables de comprendre et de résoudre les nombreux nouveaux problèmes auxquels ils seront confrontés. C'est pourquoi l'enquête PISA 2012 avait évalué les compétences des élèves en résolution individuelle de problèmes.

La capacité à résoudre individuellement des problèmes non familiers est certes essentielle, mais dans notre monde de plus en plus interconnecté, nous sommes souvent amenés à collaborer pour atteindre nos objectifs, tant dans notre vie professionnelle que privée. Le travail en équipe présente de nombreux avantages : il permet de disposer de tout un éventail de points de vue et d'expériences ; des synergies peuvent se créer entre les membres du groupe, ce qui permet d'améliorer la qualité et l'efficacité des solutions ; et enfin, il est possible de procéder à une division du travail et à une répartition des tâches en fonction des points forts de chacun.

La collaboration peut toutefois aussi présenter son lot de difficultés. Si la répartition des tâches n'est pas efficace, deux membres du groupe peuvent se retrouver à effectuer le même travail. Des tensions interpersonnelles et une mauvaise communication au sein du groupe peuvent également l'empêcher d'exploiter pleinement son potentiel. Si elles ne sont peutêtre pas innées chez tous, les compétences de collaboration peuvent néanmoins se développer avec le temps et la pratique.

C'est pourquoi l'enquête PISA 2015 a décidé d'étendre son investigation au-delà de la résolution individuelle de problèmes et de mesurer - pour la toute première fois dans une évaluation internationale - la capacité des élèves à collaborer pour la résolution de problèmes. Les élèves de 52 systèmes d'éducation se sont prêtés à cette évaluation.

\section{Qu'entend-on par compétences en résolution collaborative de problèmes ?}

Xandar, l'une des six unités utilisées dans le cadre de cette évaluation informatisée, a été rendue publique. Dans cette dernière, l'élève évalué fait partie d'une équipe (point développé ci-après) invitée à répondre, sur le modèle d'un concours de quiz, à une série de questions sur le pays fictif de Xandar.

En 2012, PISA avait identifié quatre processus dans le cadre de la résolution individuelle de problèmes :

- la collecte d'informations liées au problème

- la représentation du problème et de ses différentes relations à l'aide de tableaux, de graphiques, de symboles ou de mots

- la planification et l'exécution d'une stratégie pour résoudre le problème

- le suivi de la bonne mise en œuvre de cette stratégie et la réaction au feedback reçu durant le processus de résolution du problème.

Ces quatre processus restent pertinents pour la dimension de résolution de problèmes de l'évaluation PISA 2015, mais sont complétés par trois compétences spécifiques à la résolution collaborative de problèmes :

- établir et maintenir une compréhension commune (découvrir ce que savent les autres membres du groupe et veiller à ce que tous partagent la même vision du problème)

- entreprendre les actions adéquates pour résoudre le problème (déterminer les actions collaboratives à mettre en œuvre - par exemple, « qui fait quoi ?»-, puis les exécuter) 
- établir et maintenir une organisation de groupe (assurer le suivi de son propre rôle dans la stratégie de résolution de problèmes et vérifier que les autres s'acquittent du rôle qui leur a été confié).

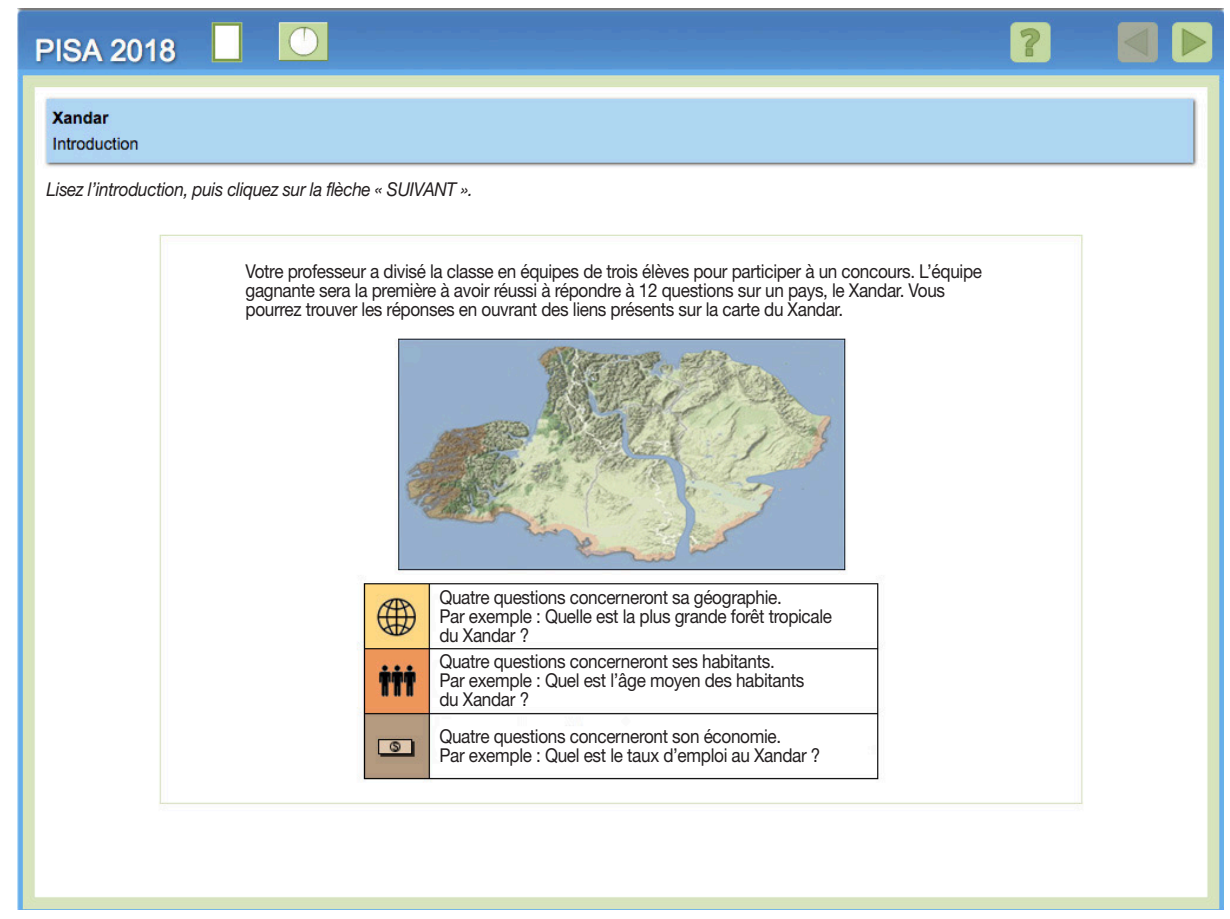

La combinaison des quatre processus de résolution individuelle de problèmes et des trois compétences de résolution collaborative de problèmes permet d'identifier 12 compétences spécifiques. Chaque item de l'évaluation de la résolution collaborative de problèmes mesure au moins l'une d'entre elles.

Prenons, à titre d'exemple, les items de l'unité Xandar:

- Dans la tâche 1 de l'item 2, l'un des membres de l'équipe souhaite discuter de la façon d'aborder le concours, tandis qu'un autre veut commencer immédiatement. Un élève évalué qui, en accord avec le premier, souhaiterait d'abord discuter de la stratégie à adopter obtiendrait un crédit pour cette réponse, car il « planifie une stratégie " pour résoudre le problème, tout en « établissant et en maintenant une compréhension commune ».

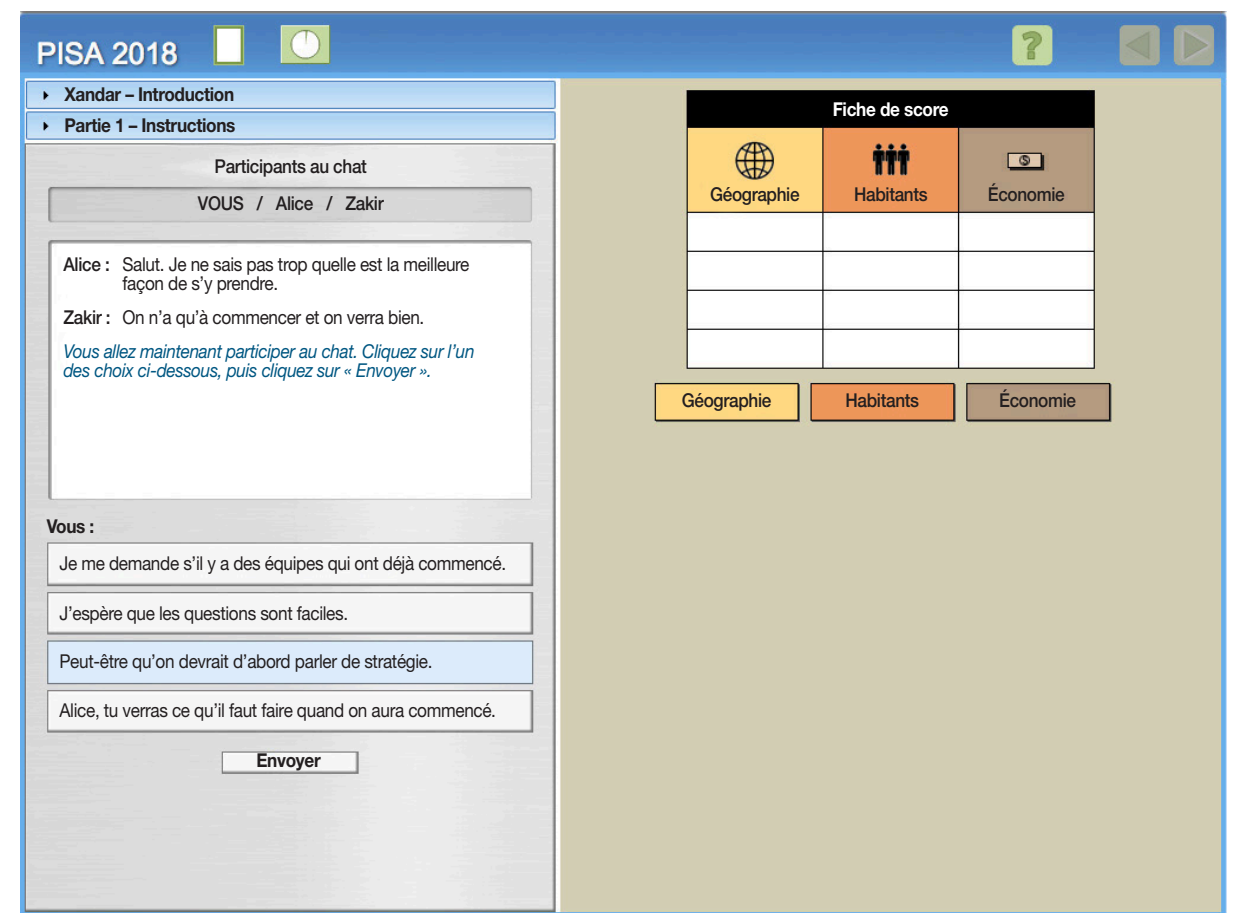


- Dans la tâche 2 de l'item 2, après l'adoption d'une stratégie prévoyant que chaque membre de l'équipe répondra aux questions de l'un des trois sujets proposés, deux membres de l'équipe se disputent le même sujet. L'un justifie mieux son souhait que l'autre. L'élève évalué doit alors lui confier les questions de ce sujet. Ce faisant, l'élève « représente le problème » et « établit et maintient une organisation de groupe ».

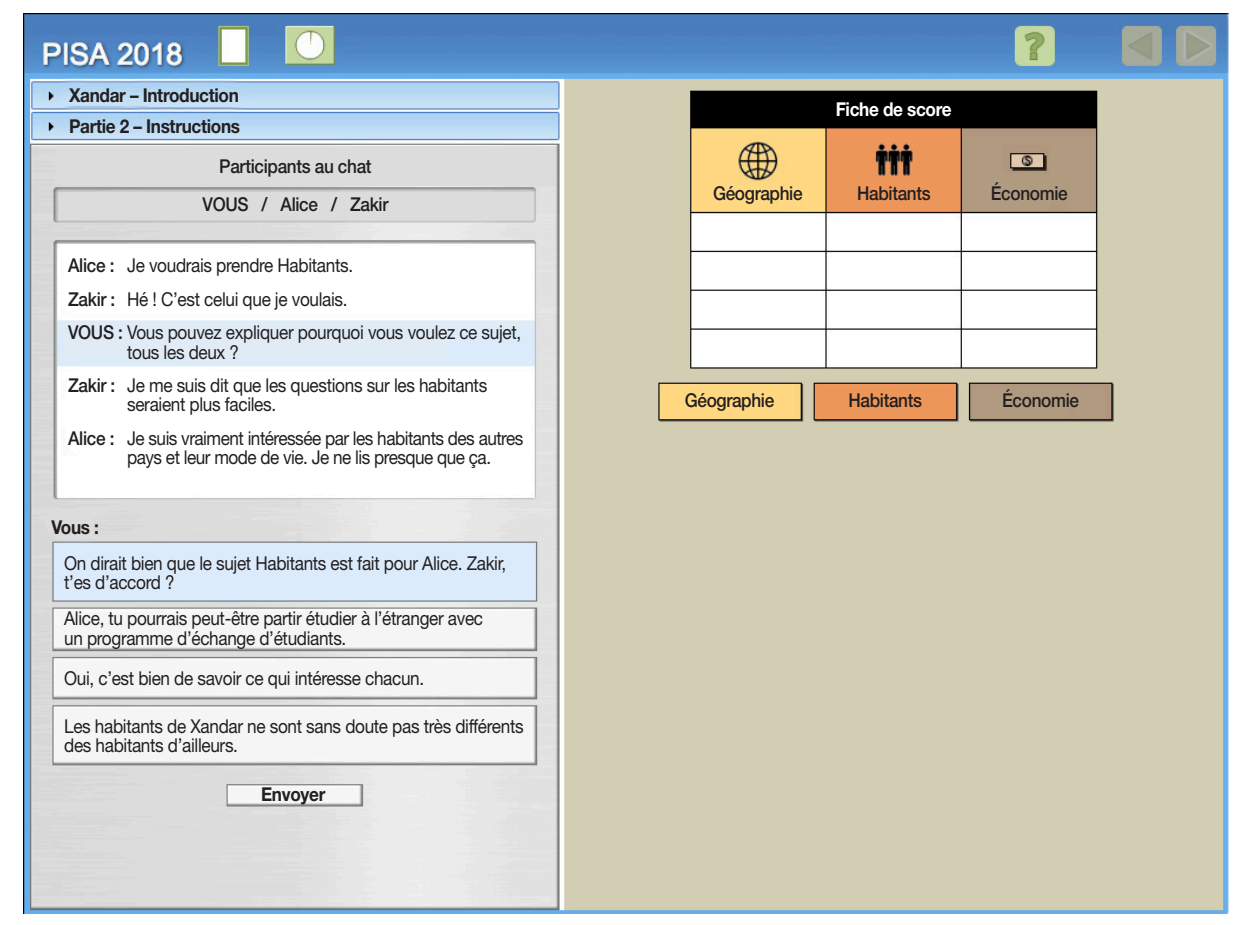

- Dans la tâche 4 de l'item 2, l'élève évalué doit consulter la fiche de score et se rendre compte que l'équipe a pris du retard sur les questions de l'un des sujets. S'il procède ainsi, il montre alors sa capacité à " assurer le suivi de la bonne mise en œuvre de la stratégie » et à « entreprendre les actions adéquates pour résoudre le problème ».

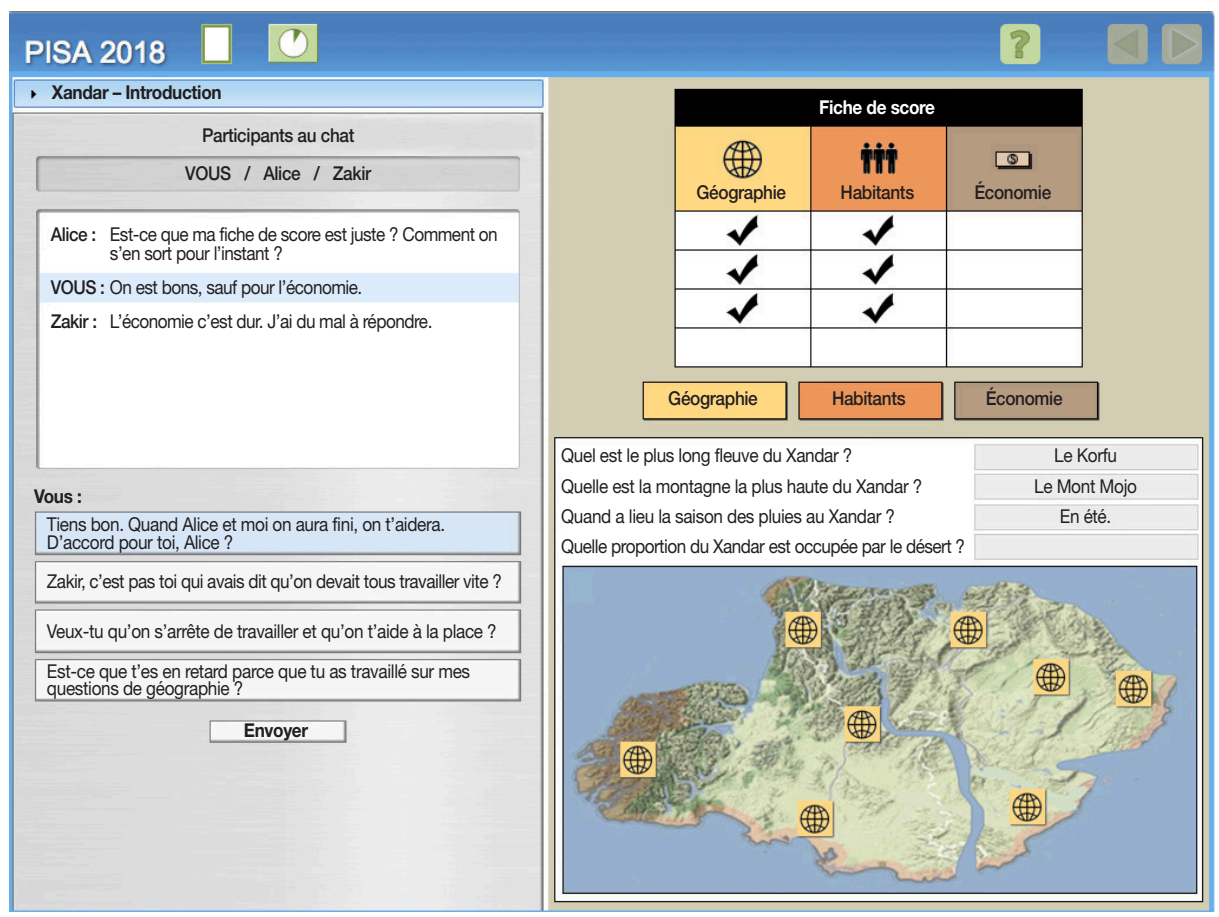




\section{Avec qui les élèves collaborent-ils dans cette évaluation?}

Dans la vraie vie, nous communiquons en général les uns avec les autres, de plus en plus via nos ordinateurs ou nos téléphones. Afin de fournir une mesure précise des compétences des élèves en résolution collaborative de problèmes c'est-à-dire non dépendante de la performance de leurs co-équipiers -, l'enquête PISA a mis à profit l'informatisation des épreuves pour faire interagir les élèves évalués avec des simulations informatisées d'humains (aussi appelées « agents virtuels ») dont le comportement peut être contrôlé. Quel que soit la réponse de l'élève évalué, ces agents virtuels ramènent le problème au même point. Les élèves sont ainsi confrontés à la même série de décisions indépendamment de leurs actions antérieures. En outre, les agents virtuels peuvent être programmés de sorte que certains se montrent plus coopératifs ou plus concentrés sur la résolution du problème que d'autres - tout comme pourraient le faire de vraies personnes.

Mais les interactions humain-machine donnent-elles une idée fiable des interactions de personne à personne ? Voici les conclusions d'une étude commandée par l'OCDE à ce sujet :

- Aucune différence pertinente d'un point de vue pratique ne s'observe dans les réponses des élèves à deux versions des tâches de résolution collaborative de problèmes : la version informatisée originale et une version reformatée dans laquelle l'agent virtuel est remplacé par une personne qui ne peut choisir ses réponses que parmi un choix limité d'options.

- Une bonne corrélation s'observe entre l'avis des enseignants sur les compétences de collaboration de leurs élèves et la performance de ces derniers à l'évaluation (informatisée) de la résolution collaborative de problèmes.

- Certains élèves ont effectué plusieurs tâches de résolution collaborative de problèmes avec un agent virtuel, puis deux tâches en face à face avec une autre personne réelle, dans lesquelles ils avaient la possibilité d'interagir librement, et non via un ensemble limité d'options à choix multiple. Leur performance avec l'agent virtuel s'avère une variable prédictive relativement probante de leur performance avec le co-équipier humain.

Par conséquent, bien que la façon dont les élèves collaborent diffère selon qu'ils travaillent avec un agent virtuel ou une personne réelle, l'évaluation informatisée utilisée dans le cadre de l'enquête PISA permet tout de même de donner un aperçu de la capacité des élèves à collaborer avec autrui.

\section{Pour conclure}

Alors, qu'attendre fin novembre de la publication des résultats de l'évaluation de la résolution collaborative de problèmes?

Entre autres :

- Quel pays/économie obtient le meilleur score moyen en résolution collaborative de problèmes?

- Comme dans l'enquête PISA 2012, il est probable qu'une relation positive s'observe entre, d'un côté, la performance en sciences, en compréhension de l'écrit et en mathématiques, et de l'autre, celle en résolution collaborative de problèmes. Si l'on compare des élèves de différents systèmes d'éducation ayant un niveau de compétence similaire dans les trois principaux domaines d'évaluation PISA, dans quels systèmes les élèves obtiennent-ils la meilleure performance en résolution collaborative de problèmes ?

- Qui des garçons ou des filles réussit le mieux en résolution collaborative de problèmes ?

- Quelles sont les attitudes des élèves à l'égard de la collaboration ?

- Certains comportements des élèves ou certaines politiques des établissements sont-ils associés à une meilleure performance en résolution collaborative de problèmes ou à des attitudes plus positives à l'égard de la coopération ? 


\section{Pour tout complément d'information}

Contacter : Jeffrey Mo (jeffrey.mo@oecd.org)

Le mois prochain : Les élèves savent-ils comment collaborer pour la résolution de problèmes ?

Cet ouvrage est publié sous la responsabilité du Secrétaire général de l'OCDE. Les opinions et les interprétations exprimées ne reflètent pas nécessairement les vues des pays membres de l'OCDE.

Ce document, ainsi que les données et cartes qu'il peut comprendre, sont sans préjudice du statut de tout territoire, de la souveraineté s'exerçant sur ce dernier, du tracé des frontières et limites internationales, et du nom de tout territoire, ville ou région.

Ce texte est disponible sous licence Attribution - Pas d'Utilisation Commerciale - Partage dans les Mêmes Conditions 3.0 Organisations Internationales (CC BY-NC-SA 3.0 IGO). Pour toute information spécifique quant à l'étendue et aux termes de la licence ainsi que d'une possible utilisation commercial de ce texte or pour toute usage de données PISA, prière de consulter les Conditions d'utilisation à http://www.oecd.org/fr/conditionsdutilisation. 